\title{
Dynamical Study of the Exoplanet Host Binary System HD 106515
}

\author{
F. M. Rica ${ }^{1,7}$, R. Barrena ${ }^{2,3}$, J. A. Henríquez ${ }^{4,5}$, F. M. Pérez ${ }^{6}$ and P. $\operatorname{Vargas}^{6}$ \\ ${ }^{1}$ Astronomical Federation of Extremadura, C/José Martínez Ruíz Azorín, 14, 4 D, Mérida E-06800, Spain \\ ${ }^{2}$ Instituto de Astrofísica de Canarias, C/ Vía Láctea, s/n, La Laguna E-38205, Spain \\ ${ }^{3}$ Departamento de Astrofísica, Universidad de La Laguna, C/ Astrofísico Francisco Sánchez, s/n, La Laguna E-38201, Spain \\ ${ }^{4}$ Grupo de Observadores Astronómicos de Tenerife (GOAT), Spain \\ ${ }^{5}$ La Palma Astronomical Society, C/ A, no 2, Breña Baja E-38712, Spain \\ ${ }^{6}$ Universidad de La Laguna, C/ Astrofísico Francisco Sánchez, s/n, La Laguna E-38201, Spain \\ ${ }^{7}$ Email: frica0@gmail.com
}

(RECEIVED March 28, 2016; ACCEPTED November 22, 2016)

\begin{abstract}
HD 106515 AB (STF1619 AB) is a high common proper motion and common radial velocity binary star system composed of two G-type bright stars located at $35 \mathrm{pc}$ and separated by about 7 arcsec. This system was observed by the Hipparcos satellite with a precision in distance and proper motion of 3 and $2 \%$, respectively. The system includes a circumprimary planet of nearly 10 Jupiter masses and a semimajor axis of $4.59 \mathrm{AU}$, discovered using the radial velocity method. The observational arc of $21^{\circ}$ shows a small curvature that evidences HD $106515 \mathrm{AB}$ is a gravitationally bound system. This work determines the dynamical parameters for this system which reinforce the bound status of both stellar components. We determine orbital solutions from instantaneous position and velocity vectors. In addition, we provide a very preliminary orbital solution and a distribution of the orbital parameters, obtained from the line of sight $(z)$. Our results show that HD $106515 \mathrm{AB}$ presents an orbital period of about 4800 years, a semimajor axis of $345 \mathrm{AU}$ and an eccentricity of about 0.42 . Finally, we use an N-body numerical code to perform simulations and reproduce the longer term octupole perturbations on the inner orbit.
\end{abstract}

Keywords: (stars:) binaries: visual, stars: individual: (HD 106515), stars: kinematics and dynamics, (stars:) planetary systems

\section{INTRODUCTION}

To date, more than 3400 exoplanets in about 2570 planetary systems have been discovered using different observational techniques. About $10 \%$ of these planets are found in stellar systems with two or more stars (Adams et al. 2012; Roell et al. 2012; Lodieu et al. 2014). The stellar multiplicity can alter the dynamical evolution and the orbit of the planets due to the Kozai-Lidov mechanism [hereafter KL; see Kozai (1962) and Lidov (1962)] and other phenomena. The KL is a dynamical perturbation in the orbit of a satellite (planet or star) by the gravitational influence of another body on a wider and misaligned orbit. Astronomers are now able to study the differences in the planetary orbital parameters and masses between single and multiple stellar systems. The dynamical study of these stellar systems can shed some light on the possible values for their orbital parameters, increasing the knowledge about exoplanet formation and evolution. For example, the orbital inclination allows us to conclude if the $\mathrm{KL}$ is the cause of the high orbital eccentricity observed in planets around stars in multiple systems and the periodic change of the planetary orbital parameters. The eccentricity and semimajor axis allow us to calculate the boundaries of stability within a three body system, which are important parameters to define the orbital planetary stability and stable zones around a stellar component. Hauser \& Marcy (1999) studied dynamically the stellar system 16 Cygni analysing the relative astrometry and the orbital parameters for a bound condition. They concluded that the wide stellar component is the cause of the high planetary eccentricity.

GAIA (Global Astrometric Interferometer for Astrophysics) is a European Space Agency (ESA) space astrometry mission launched on 2013 December 19. It is the successor of Hipparcos satellite and will monitor about 1 billion objects down to magnitude 20 , during its 5-yr mission. It will provide distances, positions and proper motions with an unprecedented accuracy of 0.02 and 0.20 mas for bright and faint stars (mag $\leq 19$ ), respectively, as well as radial velocities. GAIA will determine the distances to the nearest objects 
with accuracy of $0.001 \%$. This extremely accurate astrometric and kinematical data will be a great help in determining orbital parameters of wide binaries using a suitable orbital calculation method and different from those used for close visual binaries. While waiting for GAIA data, it is important to perform dynamical studies and to determine orbital solutions distribution for binary stars hosting exoplanets improving techniques that make use of data obtained from the Earth.

GAIA data can be exploited using the Apparent Motion Parameter (Kiselev \& Kiyaeva 1980; Kiselev 1989), hereafter AMP, or Hauser \& Marcy (1999) method for orbital estimations. These techniques only need very accurate instantaneous position $(x, y, z)$ and velocity vectors $\left(V_{x}, V_{y}, V_{z}\right)$ in addition to the masses of the stellar components and a trigonometric parallax.

HD 106515 AB (WDS 12151-0715 AB = STF1619 AB $=$ HIP 59743) is a wide binary system that is astrometrically well-sampled and has a high common proper motion (hereafter CPM) which was first observed by de Lalande (1831) in 1795. The system is composed of two stars G5/6V and G8/9V with $V=7.96$ and $V=8.22 \mathrm{mag}$, respectively. Since 1795 , it has been observed 135 times. During this time, the angular separation and position angle has changed from 8.0 to 6.9 arcsec and from $295^{\circ}$ to $266^{\circ}$, respectively. Gould \& Chanamé (2004) and Marmier et al. (2013) based only on the CMP concluded that HD 106515 is composed of stars gravitationally bound. The common radial velocity (RV hereafter) was used by Desidera et al. (2012) to reinforce the bound status. These are necessary, but in same cases not sufficient conditions. In this work, we add new important evidence (small curvature, common distance, dynamic stability, etc.) to assure the gravitationally bound status.

A planet of nearly 9.6 Jupiter masses was discovered around HD 106515 A by measuring radial velocities (Mayor et al. 2011), with an estimated orbital period of $3630 \mathrm{~d}$ (about $10 \mathrm{yrs}$ ), which is one of the largest orbital periods for planets discovered using RV techniques. Desidera et al. (2012) studied the binary system determining orbital parameters, which allowed them to define the critical semimajor axis as a function of $z$ (and therefore the stable zones around the stars). They suggested that the high eccentricity of the planet could be caused by KL.

In this work, we developed a dynamical and orbital study more complete and detailed than in the literature, tabulating complete positional and dynamical parameters in addition to same orbital solutions with the corresponding orbital plot. We also study the dynamical evolution of the HD 106515 b orbit using, for the first time in this system, an N-body numerical code to simulate and reproduce the longer term octupole perturbations, the so-called Eccentric Kozai-Lidov mechanism (hereafter EKLM).

The Washington Double Star Catalog (Mason et al. 2001), hereafter WDS, centralises all the astrometric measurements for visual double stars, includes a third stellar component (listed as C) with $10.5 \mathrm{~V}$ magnitude and low proper motion at an angular separation of 98 arsec to the HD 106515 A. Marmier et al. (2013) concluded no probable physical relation to the HD 106515 AC system based on the very different proper motions of $\mathrm{C}$ and $\mathrm{AB}$. We add a new astrometric measure.

In this work, our main aim is to improve the orbital solution of HD $106515 \mathrm{AB}$ with respect to that determined in Desidera et al. (2012) by using a method similar to that proposed by Hauser \& Marcy (1999). The astrophysical properties estimates performed in this work follow the guidelines detailed in Benavides et al. (2010) and Rica (2012).

The organisation of this paper is as follows. In Section 2, we present the dynamics of $\mathrm{A}$ and $\mathrm{C}$ components and the nature of $\mathrm{C}$ is confirmed. In Section 3, we detail astrometric dataset used to compute the relative motion of $\mathrm{B}$ with respect to A component. In Section 4, we provide new arguments which support that A and B stars are gravitationally bound. The method of orbital calculation is described in Section 5, and the new orbital solutions are exposed in Section 6. Finally, we discuss the results in Section 7 and expose the conclusions in Section 8.

\section{ASTROPHYSICAL PROPERTIES OF HD 106515 A AND THE WIDE COMPONENT C}

HD $106515 \mathrm{~A}$ is a solar metallicity star of $V=7.96 \mathrm{mag}$ classified as a G5V type star (Adams et al. 1935) with a high proper motion. The distance was determined by Hipparcos satellite (reviewed values from van Leeuwen (2007): $\pi=$ $28.42 \pm 0.96$ mas; $35.2 \pm 1.2 \mathrm{pc})$. An $E(B-V)=0.01$ was obtained in this work following the procedure described in Benavides et al. (2010) and Rica (2012). Table 1 lists astrophysical data for the $\mathrm{A}$ and $\mathrm{B}$ components.

In 1910, Burnham (1913), using the refractor telescope of $1.0 \mathrm{~m}$ in the Yerkes Observatory, observed a star of 9.5 mag (listed in UCAC4 catalogue with $V=10.49 \mathrm{mag}$ ) at $99 \operatorname{arcsec}$ and with direction $177^{\circ}$ to HD 106515 A. It is listed in the WDS catalogue as the $\mathrm{C}$ component, with a small proper motion and therefore it is a background unbound star. Marmier et al. (2013) already noted the great difference in proper motion and concluded that $\mathrm{C}$ component is "probably not physical'.

In this work, we confirm the great relative motion between the $\mathrm{A}$ and $\mathrm{C}$ components. Today, there are 10 astrometric measures in the literature, the last one in 2014 , performed by Nuget \& Iverson (2015) using the video drift method. By using the historical relative astrometries with a base line of $121 \mathrm{yrs}$, we show the change of the relative motion of $\mathrm{C}$ component with respect to the primary component. These astrometries are listed in the WDS catalogue. We add a new astrometric point for 2010.5589 from WISE catalogue (162.93 and 99.08 arcsec). We obtain a relative motion in $R A=$ $+240.3 \pm 4.8$ mas yr$^{-1}$ and $D E C=+43.3 \pm 1.1$ mas yr$^{-1}$, yielding a proper motion for $\mathrm{C}\left(\mu(\alpha)=-9.8 \pm 4.9 \mathrm{mas} \mathrm{yr}^{-1}\right.$ and $\mu(\delta)=-9.6 \pm 1.6$ mas $\left.^{-1}\right)$, which is in agreement 
Table 1. Astrophysical data for components of HD 106515 AB.

\begin{tabular}{|c|c|c|c|}
\hline & Primary & Secondary & Reference \\
\hline$\alpha_{2000}$ & \multicolumn{2}{|c|}{$12 \mathrm{~h} 15 \mathrm{~m} 06.57 \mathrm{~s}$} & \\
\hline$\delta_{2000}$ & \multicolumn{2}{|c|}{$-07^{\circ} 15^{\prime} 26.4^{\prime \prime}$} & \\
\hline$V^{a}$ & +7.99 & +8.25 & This work \\
\hline$B-V$ & \multicolumn{2}{|c|}{$+0.815 \pm 0.003$} & Hipparcos \\
\hline$V-I$ & \multicolumn{2}{|c|}{$+0.83 \pm 0.02$} & Hipparcos \\
\hline$J$ & \multicolumn{2}{|c|}{$+6.585 \pm 0.017$} & 2MASS \\
\hline$H$ & \multicolumn{2}{|c|}{$+6.218 \pm 0.043$} & 2MASS \\
\hline$K$ & \multicolumn{2}{|c|}{$+6.151 \pm 0.021$} & 2MASS \\
\hline$\mu(\alpha)\left(\operatorname{mas}_{\mathrm{yr}}-1\right)$ & $-250.1 \pm 1.2$ & $-240.8 \pm 1.0$ & Tycho-2 Hog et al. (2000) \\
\hline$\mu(\delta)\left(\operatorname{mas~yr}^{-1}\right)$ & $-52.9 \pm 1.2$ & $-69.4 \pm 1.0$ & Tycho-2 Hog et al. (2000) \\
\hline \multirow{3}{*}{ Spectral type } & G5V & G8V & Adams et al. (1935) \\
\hline & KOIV & K0/1IV & Houk \& Swift (1999) \\
\hline & K0V & K1V & This work \\
\hline Distance $(\mathrm{pc})$ & \multicolumn{2}{|c|}{$35.19 \pm 0.74$} & van Leeuwen (2007) \\
\hline Radial Velocity $\left(\mathrm{km} \mathrm{s}^{-1}\right)$ & $20.66 \pm 0.11$ & $19.94 \pm 0.11$ & Desidera et al. (2006) \\
\hline \multirow[t]{3}{*}[\mathrm{Fe}/\mathrm{H}]{} & $0.08 \pm 0.066$ & $0.06 \pm 0.062$ & Desidera et al. (2004) \\
\hline & $0.03 \pm 0.02$ & $\ldots$ & Santos et al. (2013) \\
\hline & 0.01 & 0.00 & Desidera et al. (2006) \\
\hline \multirow[t]{2}{*}{ Mass $\left(\mathrm{M}_{\odot}\right)$} & $0.88 \pm 0.06$ & $\ldots$ & Santos et al. (2013) \\
\hline & $0.91 \pm 0.03$ & $0.88 \pm 0.03$ & Desidera et al. (2012) \\
\hline Age (Gyr) & $6 \pm 2$ & $\ldots$ & Desidera et al. (2012) \\
\hline
\end{tabular}

a Values obtained in this work, converting the Hipparcos magnitudes to $V$ band. The conversion was performed in an iterative process. We compute the spectral type from the initial $H_{p}$ magnitudes and the parallax, which gives the correction $\mathrm{Hp}-\mathrm{V}$ for the magnitude. Using these new magnitudes, the process is repeated until the iteration converges into a fix value.

with the values present in Tycho-2 catalogue. This clearly confirm that the $\mathrm{C}$ component is a background star.

By using the reduced proper motion diagram of Jones (1972), photometric data (APASS, 2MASS) and the proper motion (Tycho-2), we suggest an evolved nature for the $\mathrm{C}$ component.

We use a CMD (Colour Magnitude Diagram) 2.5 evolutionary isochrones ${ }^{1}$ (we assumed solar metallicity) to determine a spectral type of K0III-IV, which is in excellent agreement with Pickles \& Depagne (2010) who also determined a distance of $246 \mathrm{pc}$ by using only photometric data. In addition to the spectral type, we also determine $M v=2.5_{-0.9}^{+0.5}$ mag and distance of $370_{-65}^{+205} \mathrm{pc}$. The centroid values correspond with solar metallicity and age (metalliticy and age for $\mathrm{C}$ component are not listed in the literature). The errors correspond to ages of 2 and 8 Gyr. Therefore, the photometric distance for $\mathrm{C}$ is much larger than that corresponding to the $\mathrm{AB}$ pair. This is new evidence that reinforces the unbound nature of $\mathrm{C}$ with respect to the $\mathrm{AB}$ system.

\section{ASTROMETRIC DATA AND DYNAMICAL STUDY OF HD 106515 AB}

In 1795, de Lalande found a bright star at 8.01 arcsec from HD 106515 with position angle $294.5^{\circ}$. Twenty-eight years later, in 1823, South \& Herschel (1824) measured this pair

${ }^{1} \mathrm{http} / / /$ stev.oapd.inaf.it/cgi-bin/cmd, using PARSEC isochrones (Bressan et al. 2012) version 1.1 with the photometric system UBVRIJHK (Bessell 1990; Maíz-Apellániz 2006). again $\left(288^{\circ}\right.$ and $\left.9.23 \operatorname{arcsec}\right)$. Three years later, Struve (1837) performed a third measure and estimated visual magnitudes of 7.5 and 7.8 for the stellar components. The Struve designation was finally assigned to this double star.

Since its discovery, this pair of stars has been measured 135 times, the last one in 2014 by non-professional astronomers, see Nuget \& Iverson (2015), using video drift method. There are many micrometrical measurements but also many other accurate photographic captures. The position angle has decreased from $288^{\circ}$ to $266^{\circ}$ and the angular separation has changed from 7.9 to 6.9 arcsec. Brian Mason (US Naval Observatory) kindly supplied astrometric information for HD $106515 \mathrm{AB}$.

The WDS catalogue lists several micrometric measures taken with 0.7-m telescopes (with a Rayleigh resolution limit of $0.19 \mathrm{arcsec}^{2}$. Only one high-resolution observation was performed with a telescope of aperture greater than $0.7 \mathrm{~m}$. In 1910, Burnham used the 1.0-m telescope of Yerkes Observatory (with a Rayleigh resolution limit of 0.14 arcsec) in very good sky conditions. HD 106515 A and B have never been observed using digital high-resolution technique (lucky imaging, speckle, or adaptive optics) with a telescope using an aperture greater than $1.0 \mathrm{~m}$.

\footnotetext{
${ }^{2}$ These $0.7-\mathrm{m}$ telescopes were located at the US Naval Observatory (USA), Royal Observatory of Greenwich (UK), McCormick Observatory (USA), Observatory of Johannesburg (South African) and the Observatory of the Viena University (Austria, Europe)
} 


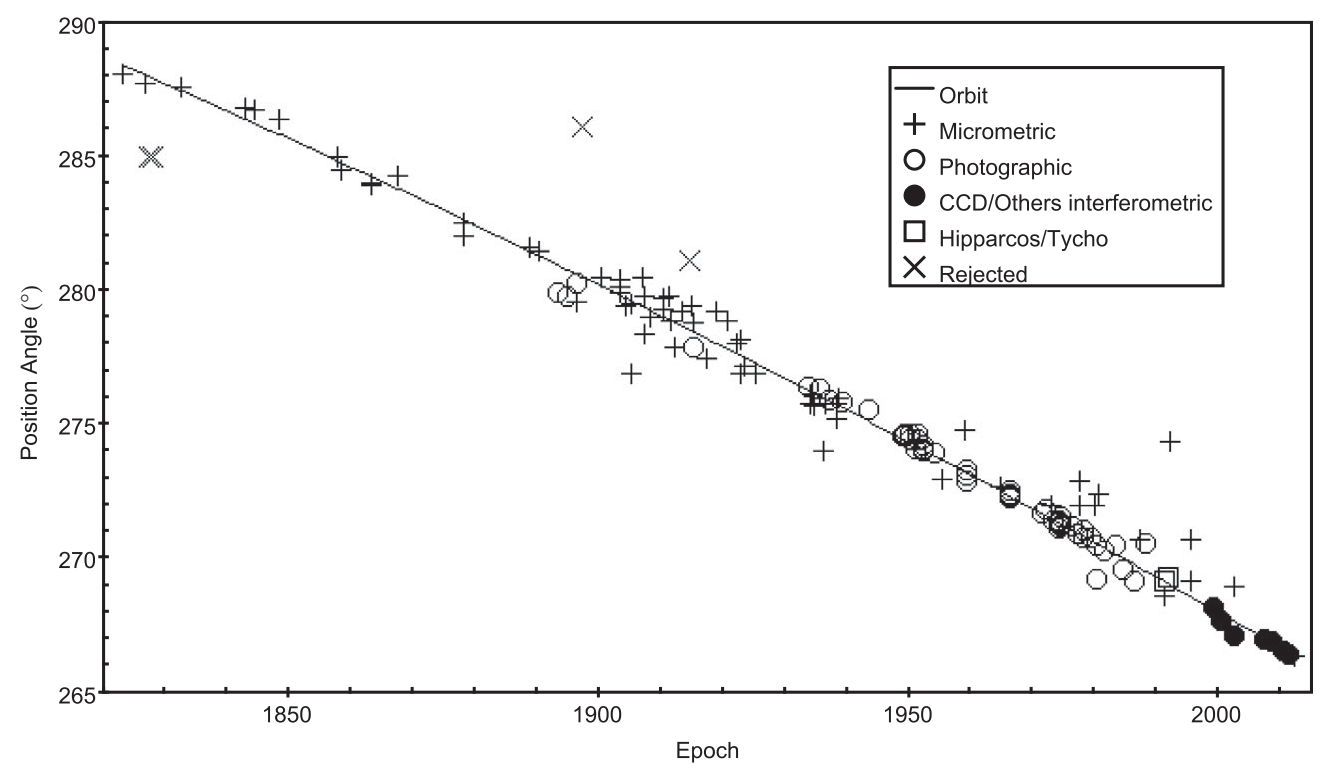

Figure 1. The weighted linear fit of the position angle $(\theta)$ determines a $\mathrm{d} \theta / \mathrm{d} t=-0.133 \pm 0.002{\mathrm{deg} \mathrm{yr}^{-1}}^{-}$. The solid curve is the orbit calculated in this work.

\subsection{Digital high-resolution imaging from TCS/Fastcam}

On 2015 March 15 between 01:21 and 02:41 UT, we took about 50000 short-exposure $(30 \mathrm{~ms})$ images of HD 106515 $\mathrm{A}$ and B using the FastCam 'lucky imaging' camera, that provides a low resolution limit of about 0.14 arcsec. This instrument is mounted in the 1.5-m Carlos Sánchez Telescope in the Observatorio del Teide. More information about the Fastcam camera can be found in Oscoz et al. (2008). Our main goal was to detect new close companions to the A and B components. However, no new companion was detected. The FWHM of the final combined images was of about 0.25 arcsec. So, a star as bright as the primary component could be detected at a distance of 0.20 arcsec or wider.

\subsection{Dynamical study}

We study the relative and projected motions of the secondary component respect to the primary. We perform a weighted fit to analyse the variation of position angle $(\theta$, is the position of the secondary component with respect to the primary measured in degrees from north through east) and angular distance ( $\rho$, the apparent distance between the stellar components measured in arcsecs) over the time (see Figures 1 and 2). The $\theta$ values were corrected for precession of the coordinate system. Three measures were rejected due to their large residuals Herschel $(1829,1870)$ and the measure from Heidelberg Zodiacal 50 - WFD9999. Therefore, the dynamical study presented here includes 133 positions covering a time baseline of 189 yrs. We applied a weighting procedure to estimate the orbit following prescriptions detailed in Rica et al. (2012). For $\theta$ values, the parabolic fit shows smaller RMS (root mean square) residuals than linear fit. However, $\rho$ values show no difference between parabolic and linear fits. The values obtained for $\mathrm{d} \theta / \mathrm{d} t$ (for mean epoch 2003.22)

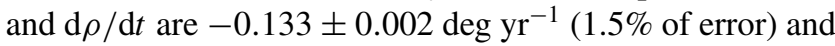
$-5.52 \pm 0.14$ mas yr $^{-1}$ (2.5\% of error), respectively.

Alternately, we compute the projected relative motion following the expression:

$$
\Delta \mu=\sqrt{\left(\rho \frac{\mathrm{d} \theta}{\mathrm{d} t}\right)^{2}+\left(\frac{\mathrm{d} \rho}{\mathrm{d} t}\right)^{2}} .
$$

Desidera et al. (2012) also published similar plots to our Figures 1 and 2 but the weighting procedure and the dynamical parameters they obtained were not detailed. Nevertheless, a comparison of our plots to those presented in Desidera et al. (2012) shows no significant difference.

We find a $\Delta \mu=16.92 \pm 0.27$ mas yr$^{-1}$ (for mean epoch 2003.22), which can also be computed by analysing $\mathrm{d} x / \mathrm{d} t$ and $\mathrm{d} y / \mathrm{d} t$. A good way to transform the weights from polar coordinates into weights for Cartesian coordinates was described by van den Bos (1932). It was used in this work and follows the expressions:

$$
\frac{1}{w_{Y}}=\frac{\sin ^{2} \theta}{w_{\theta}}+\frac{\cos ^{2} \theta}{w_{\rho}} ; \frac{1}{w_{X}}=\frac{\cos ^{2} \theta}{w_{\theta}}+\frac{\sin ^{2} \theta}{w_{\rho}} .
$$

A parabolic fit for $\mathrm{d} x / \mathrm{d} t$ shows smaller RMS residuals than that for a linear fit, suggesting the stellar pair follows a curved trajectory. While for $\mathrm{d} y / \mathrm{d} t$ a linear and parabolic fits show the same residuals. The relative motion (for 2003.22) was $17.27 \pm 0.18 \mathrm{mas} \mathrm{yr}^{-1}$, in agreement with the former value within errors. These results are in good agreement with that presented by Hartkopf \& Mason (2011b), who determined a relative motion of $16.0 \pm 1.3 \mathrm{mas} \mathrm{yr}^{-1}$ for HD $106515 \mathrm{AB}$.

We also use the individual proper motion from Tycho-2 data to compute the relative motion of B with respect to A.

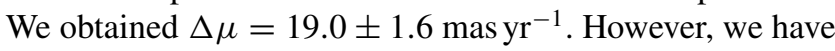




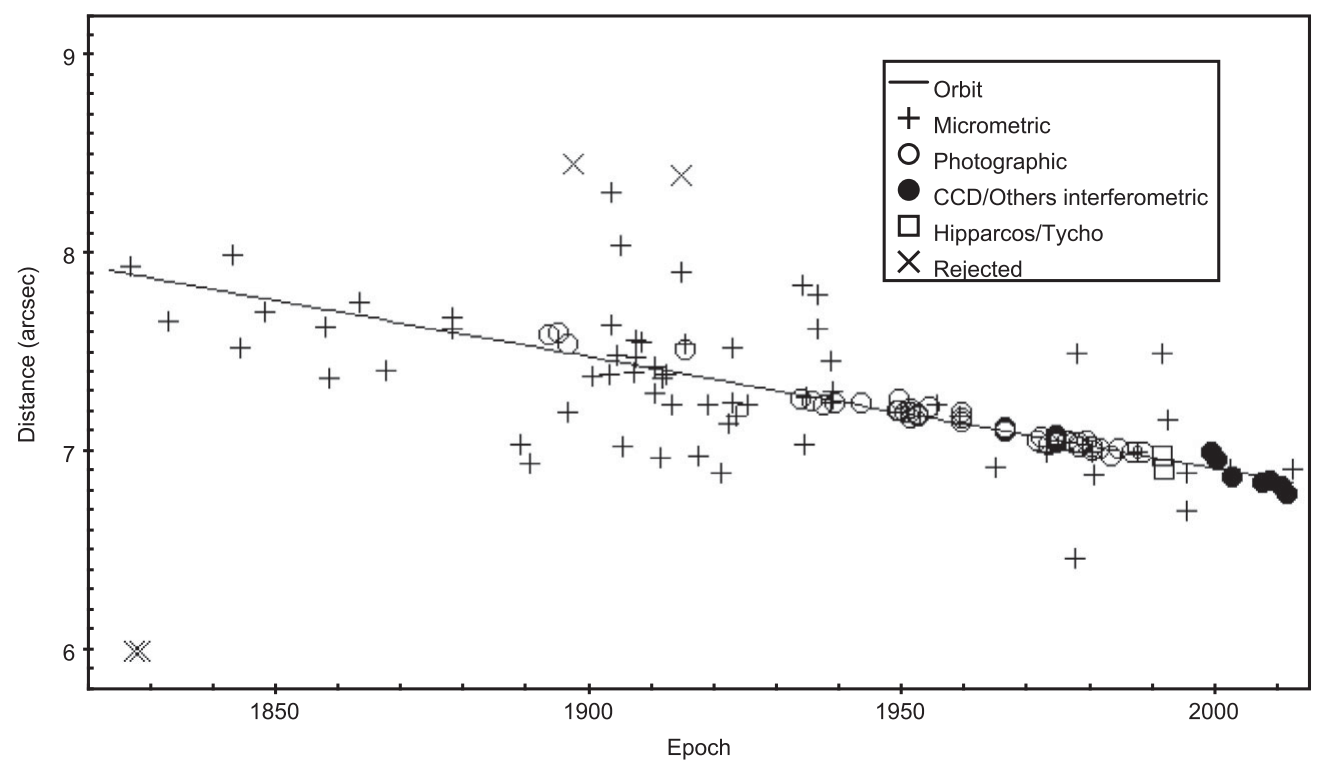

Figure 2. The same as Figure 1 but for the distance $(\rho)$. We compute a $\mathrm{d} \rho / \mathrm{d} t=-5.52 \pm 0.14 \mathrm{mas}^{-1} \mathrm{yr}^{-1}$.

to take into account that this method is often less accurate than others using a series of $\theta$ and $\rho$ values. Throughout this

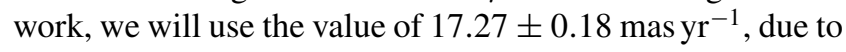
its smaller error.

We convert the relative projected motion $(\Delta \mu$ in $\operatorname{arcsec}$ $\left.\mathrm{yr}^{-1}\right)$ into the relative velocity $\left(V_{\text {rel }}\right.$, in $\left.\mathrm{km} \mathrm{s}^{-1}\right)$ at the distance $(d$, in $\mathrm{pc})$ for the system, using the expression:

$$
V_{\text {rel }}=4.74 \mathrm{~d} \Delta \mu .
$$

We obtain a relative projected velocity of $2.89 \pm 0.10$ $\mathrm{km} \mathrm{s}^{-1}$. By using the RV, for the stellar components, obtained by Desidera et al. (2006), we calculate a relative RV of $-0.72 \pm 0.16 \mathrm{~km} \mathrm{~s}^{-1}$. Thus, the total relative velocity, $V_{\text {tot }}$, is $+2.97 \pm 0.12 \mathrm{~km} \mathrm{~s}^{-1}$.

\section{PROVING THAT HD 106515 AB IS A GRAVITATIONALLY BOUND SYSTEM}

We compile solid evidence that HD 106515 AB is a gravitationally bound system. Gould \& Chanamé (2004) and Marmier et al. (2013) concluded that the system is bound based only on the CMP of the components and Gould \& Chanamé (2004), assigned the trigonometric parallax of the primary to the secondary component. CMP is necessary but not sufficient evidence to conclude the bound status of a pair of stars. It is possible that two unrelated stars show CMP by chance. Desidera et al. (2012) performed RV monitoring obtaining very accurate measurements which, added to the CMP, the common RV evidence increases the possibility to be gravitationally bound. From these two pieces of evidence, we can affirm that HD $106515 \mathrm{AB}$ travels with the same space velocity only if $\mathrm{A}$ and $\mathrm{B}$ components are at the same distance. Therefore, we need to determine if both stars are at the same distance. But even if both stars could be at common distance (within the error margins), it is not possible assure the bound status because the physical separation in line of sight is unknown (and it is possible that the relative velocity is greater than escape velocity).

In the following paragraphs, we show several proofs to demonstrate the bound status of HD $106515 \mathrm{AB}$. The proofs one and four to six are presented for the first time in this paper.

1. Curved motion. The $\mathrm{d} x / \mathrm{d} t$ and $x-y$ plots (see Figures 3 and 4) seem to show a small curvature suggesting curved orbital motion. William Hartkopf (United State Naval Observatory) concluded in private communication that, due to the small curvature shown, their linear elements Hartkopf \& Mason (2011b) must be superseded by the dynamical study provided in our paper. The linear fit for $\mathrm{d} x / \mathrm{d} t$ gives an RMS of 0.40 arcsec about $11 \%$ higher than the RMS for a non-linear fit. So, relative to this concern, a note has been added to the WDS catalogue:

A rectilinear solution to $A B$ gives a poor fit, due to slight apparent curvature suggesting very longperiod orbital motion.

Our result improves that of previous work Desidera et al. (2012).

2. The Tycho-2 catalogue lists a proper motion of $\mu(\alpha)=-250.1 \pm 1.2 \operatorname{masyr}^{-1}$ and $\mu(\delta)=-52.9 \pm$

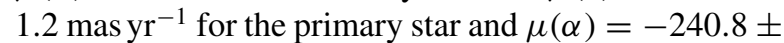
1.0 mas yr $^{-1}$ and $\mu(\delta)=-69.4 \pm 1.0$ mas yr $^{-1}$ for the secondary star. Both components have a high CPM as pointed out Gould \& Chanamé (2004) and Marmier et al. (2013).

3. Duflot, Figon, \& Meyssonnier (1995) obtained radial velocities of +21.6 and $+18.1 \mathrm{~km} \mathrm{~s}^{-1}$ for the primary and 


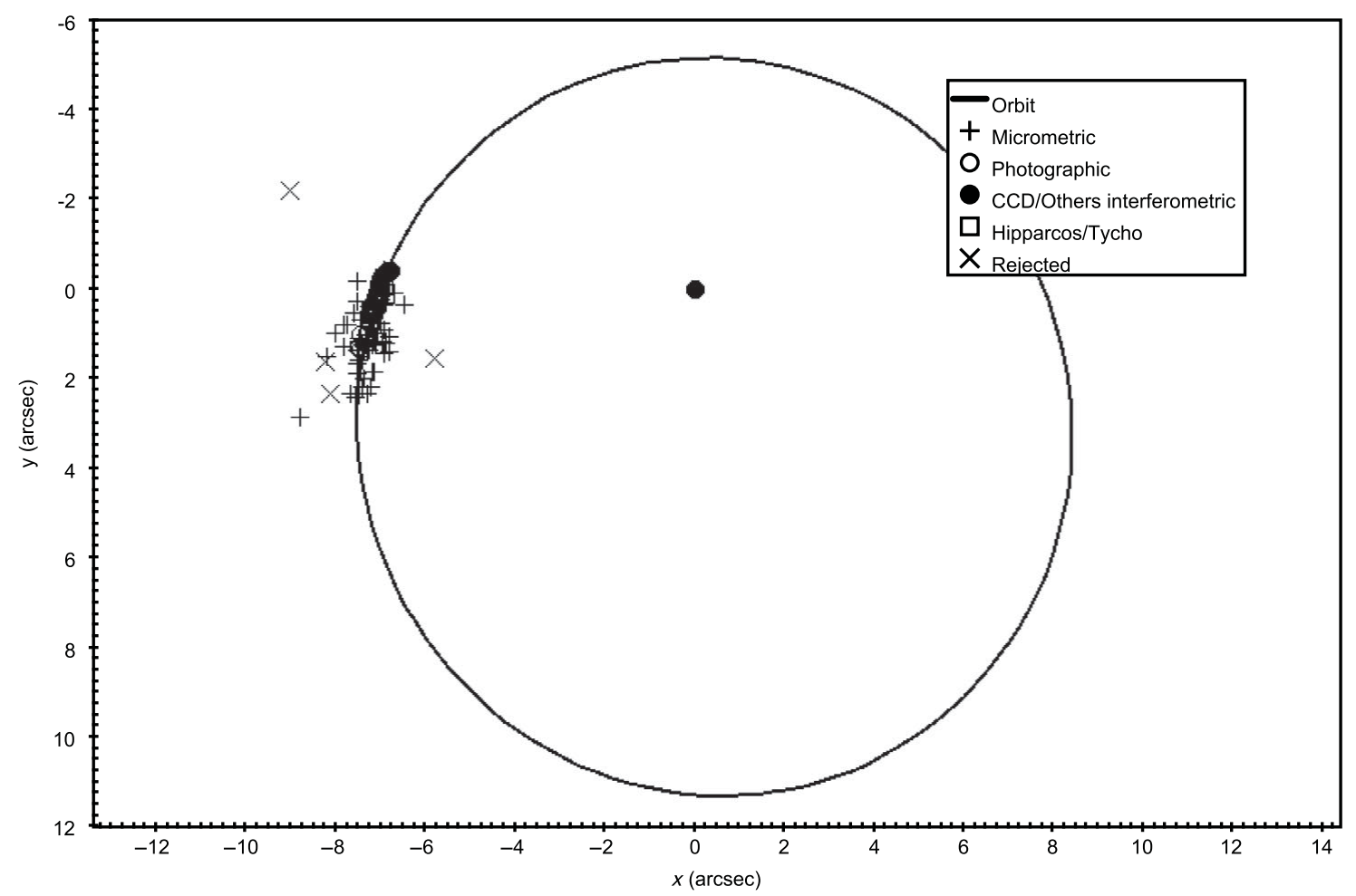

Figure 3. Orbit of HD $106515 \mathrm{AB}$ presented in this work. The line of sight separations, $z$, of A and B is of $-5.3 \mathrm{AU}$ at the epoch 2003.22. The astrometric measures are plotted as crosses, circles, and squares. The filled circle in the $(0,0)$ coordinate corresponds with the position of the primary stellar component.

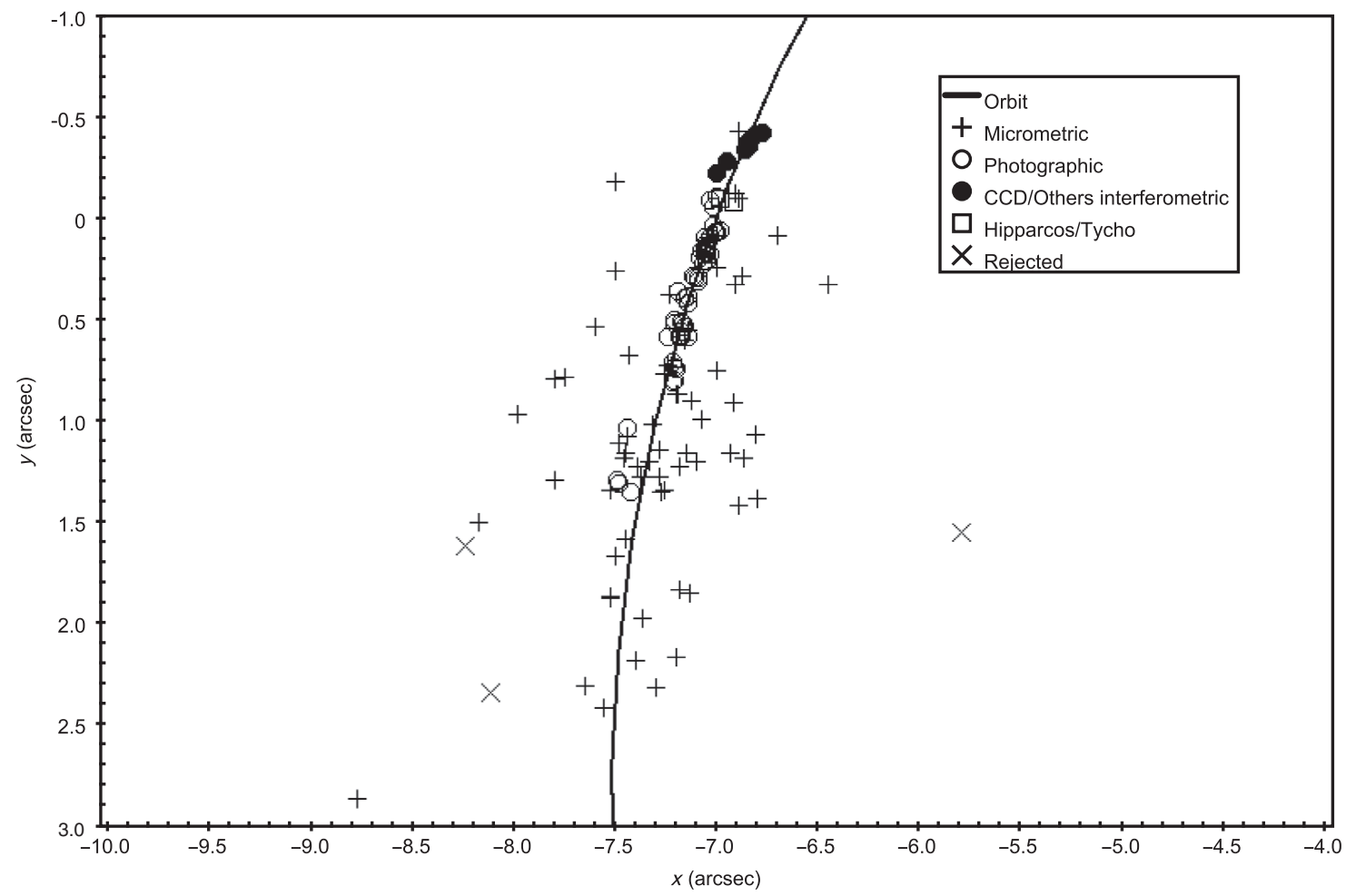

Figure 4. A zoomed in region of Figure 3. Symbols are the same as in Figure 3. 
secondary components, respectively, which are based on 27 individual measurements with a standard deviation of $1.78 \mathrm{~km} \mathrm{~s}^{-1}$. More accurate RV determinations were performed by Desidera et al. (2006) who obtained values of radial velocities for the $\mathrm{A}$ and $\mathrm{B}$ components of $+20.66 \pm 0.11$ and $+19.94 \pm 0.11 \mathrm{~km} \mathrm{~s}^{-1}$, respectively, on 2003 March 21. So, both components have a very similar RV.

4. The secondary component is classified as a G8V (Adams et al. 1935) type star with $V=8.2$. Houk \& Swift (1999) classified it as a subgiant K0/1 IV. We use an evolutionary isochrone (solar age and metallicity is assumed), accessing to a CMD 2.5 interface. Main sequence is assumed for the secondary and in the isochrone, we use Hipparcos/Tycho-2 data. If the secondary component is on the main sequence then both stars are on the same isochrone and therefore both stars present common distances. However, we can consider that there is still a remote possibility that HD $106515 \mathrm{~B}$ is a distant giant star with CPM and RV. However, Desidera et al. (2006) determined $\log (g)$ of 4.31 and 4.32 for the primary and secondary components, respectively, which are typical values of stars on the main sequence and with colours as those listed in the Tycho- 2 catalogue. Therefore, the secondary member is, as assumed, a main sequence star, and so at the same distance that the primary star.

5. Celestial mechanics is commonly used to estimate the escape velocity of stellar system (Close, Richer, \& Crabtree 1990; Mamajek 2013) and compare it to the observed total relative velocity of star B with respect to the A. A detailed description on the escape velocity computation can be found in Rica (2011). Given the separation between $\mathrm{A}$ and $\mathrm{B}$ components, the $r$ parameter is unknown, so we use its projection over the plane of the sky, $242.5 \pm 9.2 \mathrm{AU}$. It is not possible to calculate the true value of $V_{\text {esc }}$, but only its upper limit, $V_{\text {esc-max }}$. Thus, we obtain $V_{\text {esc-max }}=3.62 \pm 0.08 \mathrm{~km} \mathrm{~s}^{-1}$. A Monte Carlo approach gives $100 \%$ simulations where $V_{\text {tot }}<V_{\text {esc-max }}$ so, from this point of view, $V_{\text {tot }}$ (calculated in Section 3 ) is smaller than $V_{\text {esc-max }}$ and HD $106515 \mathrm{AB}$ could be a gravitationally bound binary.

Could HD 106515 AB be involved in a dissolution process? During the lifetime of a wide binary, the numerous encounters with passing stars and giant molecular clouds are the most important sources of disruption (Weinberg, Shapiro, \& Wasserman 1987; Jiang \& Tremaine 2010). The Galactic tidal forces only affect very wide binaries with separations of several tens of thousands of AU. HD $106515 \mathrm{AB}$ has a projected separation of $0.0012 \mathrm{pc}$. According to Figure 6 of Weinberg et al. (1987), wide binaries with an initial semimajor axis $\mathrm{a}_{0}=2000 \mathrm{AU}(0.010$ pc), with a total mass of $1 \mathrm{M}_{\odot}$ and an age of 6-8 Gyr (comparable to the age of HD $106515 \mathrm{~A}$ ) have a survival probability of 90-95\%. In fact, HD 106515 AB has a physical projected separation about eight times smaller and a total mass nearly two times larger. This yields a binding gravitational energy about 26 times larger and a survival probability of nearly $100 \%$, which means HD $106515 \mathrm{AB}$ is insensitive to external perturbers. Therefore, in this frame, three possible scenarios can be described:

- If the stellar components were born gravitationally bound, the system will remain bound today because it is insensitive to external perturbers.

- In case the stellar components were born unbound and became bound later, the same conclusion as in the first scenario can be assumed.

- A third scenario would imply that the stellar components are unbound during all their lifetime. This situation was ruled out in this work because the secondary star would not spend much time close to HD 106515 A. In fact, considering a $V_{\text {tot }}=+2.97 \pm 0.12 \mathrm{~km} \mathrm{~s}^{-1}$, the B component would spend only 0.6 Myr within the tidal radius $(1.6 \mathrm{pc})$ of the primary star. We calculate the tidal radius of the primary star using formula (2) in Mamajek et al. (2013).

6. The statistical criterion. Grocheva \& Kiselev (1998) proposed using the real distribution of proper motions to determine the possible physical relation of a pair of stars. The probability (Prob) that a pair of stars with proper motions between that of the primary and secondary is defined as $S / N$, where $S$ is the number of stars with similar proper motions in a determined sky area, and $N$ is the total number of stars in the same area. Physical pairs were defined for Prob $<0.01$. We use the PPMXL catalogue in order to obtain Prob using a circular area with a radius of $2^{\circ}$. The total number of stars $(N)$ is 80580 . Only three stars with similar proper motion was found (and two are the stellar component of HD 106515) yielding Prob $=3.7 \times 10^{-5}$, suggesting again physical relation.

\section{ANALYSIS OF THE ORBITAL MOTION}

Since HD $106515 \mathrm{AB}$ is composed of a pair of gravitationally bound stars, it is also important to obtain for this system the orbital parameters and the distribution of possible orbital solutions.

\subsection{Constraining the semimajor axis}

We can constrain the value of the semimajor axis ( $a$ arcsec) by using a relationship between $\rho$ and $a$ arcsec. Burningham et al. (2009) used the method proposed by Torres (1999) to obtain a relation between the physical projected separation $(s)$ and semimajor axis $(a)$, both in AU. They assumed randomly distributed viewing angles (inclinations) and a uniform distribution of eccentricities ranging $0<e<1$ in order to derive a relationship of

$$
E(a)=1.10_{-0.36}^{+0.91} \mathrm{~s} .
$$


Table 2. Highest values of $a \operatorname{arcsec} / \rho$.

\begin{tabular}{llccc}
\hline \hline$a$ arcsec/ $\rho$ & Double & $i(\mathrm{deg})$ & $e$ & Epoch \\
\hline 36.74 & HO 295 AB & 88 & 0.33 & 2010 \\
21.18 & STF2912 & 89 & 0.50 & 2012 \\
11.77 & COU 79 & 104.5 & 0.68 & 2010 \\
11.61 & STF2912 & 89 & 0.50 & 2011 \\
11.61 & KUI 114 & 85 & 0.74 & 2011 \\
\hline \hline
\end{tabular}

For HD $106515 \mathrm{AB}$, the value for $a(\mathrm{AU})$ is $267_{-87}^{+221} \mathrm{AU}$. The error interval is defined as a $68 \%$ confidence level. The work of Torres (1999) studies the distribution of $a \operatorname{arcsec} / \rho$ 3 and proposes a lower limit of $0.5 \rho$ in the semimajor axes estimation. This lower limit occurs when the binary is at apastron configuration and the orbit presents an eccentricity very close to 1 . A confidence level of $90-95 \%(0.5 \rho, 3.0 \rho)$ for the semimajor axis is also found in Torres (1999).

In this work, we present an independent empirical study of the relation between $\rho$ and $a$ arcsec, analysing about 300 high-quality orbits (grade 1 and 2) extracted from the Sixth Catalog of Orbits of Visual Binary Stars Hartkopf \& Mason (2011a). For each binary, the separations corresponding to the epochs 2008-2012 were compared with the semimajor axes of the orbit. Although the most extreme $a \operatorname{arcsec} / \rho$ value was 36.74 (for HO $295 \mathrm{AB}$ ) only 1-2\% of the binaries show $a$ $\operatorname{arcsec} / \rho>10$. The highest $a \operatorname{arcsec} / \rho$ values occur in very inclined orbits, where close encounters are favoured in or near the apparent periastron (see Table 2). Other possibilities for upper limits in $a \operatorname{arcsec} / \rho$ could be very eccentric orbits with the secondary star in the apparent periastron point. Only a $1.6 \%$ of the all orbital binaries compiled shows eccentricities higher than 0.95 (0.2\% presents $e>0.99)$. For HD 106515 AB system, we obtain $s=242.5 \pm 9.2 \mathrm{AU}$ (in 2003.22). The values of semimajor axis $a$ can then be constrained to (1212425) AU with a 98-99\% confidence level (0.5-10s).

\subsection{Method of orbital calculation}

An orbit of a binary star is defined with seven parameters: period $(P)$, time of periastron passage $(T)$, eccentricity $(e)$, semimajor axis $(a(\operatorname{arcsec}))$, inclination $(i)$, argument of periastron $(\omega)$, and node $(\Omega)$. To obtain the orbital solutions, we have used a method based on that presented by Hauser \& Marcy (1999). Desidera et al. (2012) used the same method to determine the distribution of the orbital solutions for HD $106515 \mathrm{AB}$. Therefore, given the simultaneity of position and velocity vectors, we can apply Newtonian mechanics to determine the distribution of the orbital parameters.

The velocity vector is composed of $V_{x}$ and $V_{y}$ perpendicular components in the plane of the sky that are computed from the astrometric data. The velocity in the line of sight, $V_{z}$,

\footnotetext{
${ }^{3}$ The distributions of $a \operatorname{arcsec} / \rho$ and $a(\mathrm{AU}) / s$ are equivalents because $a(\mathrm{AU})$ $=D a \operatorname{arcsec}$ and $s=D \rho$, where $D$ is the distance in parsecs.
}

Table 3. Astrometric, dynamical, and velocity data for HD $106515 \mathrm{AB}$.

\begin{tabular}{|c|c|}
\hline Data & Value \\
\hline Mean epoch & 2003.220 \\
\hline$\rho(\operatorname{arcsec})$ & $6.903 \pm 0.005$ \\
\hline$\theta\left({ }^{\circ}\right)$ & $267.59 \pm 0.05$ \\
\hline$x(\mathrm{AU})$ & $-242.3 \pm 9.2$ \\
\hline$y(\mathrm{AU})$ & $-10.2 \pm 0.4$ \\
\hline $\mathrm{d} \rho / \mathrm{d} t\left(\operatorname{mas~yr}^{-1}\right)$ & $-5.52 \pm 0.14$ \\
\hline $\mathrm{d} \theta / \mathrm{d} t\left(\operatorname{deg} \mathrm{yr}^{-1}\right)$ & $-0.133 \pm 0.002$ \\
\hline $\mathrm{d} x / \mathrm{d} t\left(\operatorname{mas} \mathrm{yr}^{-1}\right)$ & $+6.98 \pm 0.38$ \\
\hline $\mathrm{d} y / \mathrm{d} t\left(\operatorname{mas} \mathrm{yr}^{-1}\right)$ & $-15.80 \pm 0.11$ \\
\hline$V_{x}\left(\mathrm{~km} \mathrm{~s}^{-1}\right)$ & $+1.16 \pm 0.08$ \\
\hline$V_{y}\left(\mathrm{~km} \mathrm{~s}^{-1}\right)$ & $-2.63 \pm 0.10$ \\
\hline$V_{z}\left(\mathrm{~km} \mathrm{~s}^{-1}\right)$ & $-0.72 \pm 0.16$ \\
\hline$V_{\mathrm{esc}-\max }\left(\mathrm{km} \mathrm{s}^{-1}\right)$ & $3.62 \pm 0.08$ \\
\hline $\mathrm{M}_{\mathrm{A}}\left(\mathrm{M}_{\odot}\right)$ & $0.91 \pm 0.03$ \\
\hline $\mathrm{M}_{\mathrm{B}}\left(\mathrm{M}_{\odot}\right)$ & $0.88 \pm 0.03$ \\
\hline Distance $(\mathrm{pc})$ & $35.2 \pm 1.3$ \\
\hline
\end{tabular}

arises from the differential RV of the stellar members for the system. On the other hand, the position vector is made up by the $x$ and $y$ coordinates (on the plane of the sky) which are calculated from the astrometric data as well, and $z$, the component in the line of sight.

The $z$ parameter can be constrained following the procedure proposed by Hauser \& Marcy (1999) for bound systems. Further constraint on $z$ is possible if the observed orbital arc shows a curvature. In such cases, the radius of the curvature $\left(\rho_{\mathrm{c}}\right)$, can be obtained following the procedure used in the AMP method. HD 106515 AB shows a very small orbital curvature that allows an estimated value for $\rho_{\mathrm{c}}$. Our result yields an $r$ value very close to $s$ and therefore the orbit of HD $106515 \mathrm{AB}$ is compatible with low inclination.

The Keplerian orbital family, defined by the range of $z$ values, was further constrained using the limits of semimajor axis values, as described in Section 5.1. The initial weights for the astrometric measures were consistently assigned following the same procedure used in the linear fit.

\section{THE ORBIT OF HD 106515 AB}

The orbital parameters of HD 106515 B relative to the A component were determined taking into account their masses (see Table 1), the relative position $(x, y, z)$ and velocity vectors $\left(V_{x}, V_{y}, V_{z}\right)$ that ensure a bound orbit.

Table 3 shows a summary of the astrometric, dynamical, and velocity data considered in the orbital calculation for 2003.22 epoch $^{4}$.

The range of $z$-values that fulfill a bound condition is $-285 \leq z \leq+285$ AU. However, if we consider the limits to the semimajor axis (see Section 5.1), the new range of $z$

\footnotetext{
${ }^{4}$ We choose the 2003.22 epoch in order to be consistent with the RV and $V$ computations.
} 
Table 4. Computed orbital parameters of HD $106515 \mathrm{AB}$, assuming $r=s(z=0), r=1.11 s$ $(z= \pm 117)$, and $r=1.38 s(z= \pm 231)$.

\begin{tabular}{lccccc}
\hline \hline Parameter & $z=-231 \mathrm{AU}$ & $-117 \mathrm{AU}$ & $0 \mathrm{AU}$ & $+117 \mathrm{AU}$ & $+231 \mathrm{AU}$ \\
\hline$P(\mathrm{yr})$ & 47494 & 7881 & 4797 & 7881 & 47494 \\
$T(\mathrm{yr})$ & 2076 & 2240 & 2379 & 2409 & 2426 \\
$e$ & 0.79 & 0.47 & 0.42 & 0.56 & 0.83 \\
$a(\operatorname{arcsec})$ & 45.3 & 13.7 & 9.8 & 13.7 & 45.3 \\
$a(\mathrm{AU})$ & 1592 & 481 & 345 & 481 & 1592 \\
$i\left(^{\circ}\right)$ & 131.7 & 147.0 & 164.9 & 153.8 & 136.4 \\
$\omega\left(^{\circ}\right)$ & 254.9 & 265.3 & 246.8 & 168.2 & 139.9 \\
$\Omega\left({ }^{\circ}\right)$ & 145.4 & 135.1 & 87.6 & 9.7 & -5.9 \\
$q(\mathrm{AU})$ & 334 & 255 & 200 & 212 & 271 \\
\hline \hline
\end{tabular}

Table 5. Orbital parameters for HD 106515 AB binary.

\begin{tabular}{lcc}
\hline \hline Parameter & Value & Error \\
\hline $\mathrm{P}(\mathrm{yr})$ & $4802.1_{-1141}^{+2397}$ & $41 \%$ \\
$\mathrm{~T}(\mathrm{yr})$ & $2376.4_{-78.9}^{+44.9}$ & $17 \%$ \\
$\mathrm{e}$ & $0.420_{-0.106}^{+0.104}$ & $58 \%$ \\
$\mathrm{a}(\operatorname{arcsec})$ & $9.822_{-1.329}^{+2.68}$ & $36 \%$ \\
$\mathrm{a}(\mathrm{AU})$ & $345_{-47}^{+95}$ & $36 \%$ \\
$\mathrm{i}(\mathrm{deg})$ & $164.45_{-11.3}^{+3.5}$ & $16 \%$ \\
$\omega(\mathrm{deg})$ & $250.50_{-14.22}^{+38.07}$ & $42 \%$ \\
$\Omega(\mathrm{deg})$ & $92.06_{-2.67}^{+45.08}$ & $10 \%$ \\
\hline \hline
\end{tabular}

is $-249 \leq z \leq+249$ AU. So, according to this interval, in Table 4, we list the orbital parameters for five values of $z$ $(-231,-117,0,+117,+231 \mathrm{AU})$ which correspond to radius vector of $r=s, 1.11 s$ (the median ratio of $r$ and $s$ ) and $1.38 s(75 \% \text { percentile })^{5}$, respectively. Table 4 also lists the periastron distance $(q)$.

We obtain a family of orbits for 1000 of different values for $z$ distributed in the range of possible values. From these orbits, we choose those showing the smallest $\chi^{2}$ residuals. Table 5 compiles the orbital solution obtained. The errors were determined following the procedure of Kiselev et al. (2012), page 532. In short, the uncertainties are computed as function only of the errors in the initial data. The last column in Table 5 shows the error due to the parallax uncertainty. Figures 3 and 4 show the final orbital solution obtained for HD $106515 \mathrm{AB}$, which yields a value of $z=-5.3 \mathrm{AU}$ at 2003.22 epoch, in agreement with $\rho_{c}$ value.

Because the binary shows a small arc, the orbital solution present here is preliminary, and so we estimate the distribution of each orbital parameter as function of $z$ (see Figure 5). A visual comparison of these distributions with the corresponding plots presented in Desidera et al. (2012) shows no significant difference. However, our distribution of the eccentricities gives slightly higher values in respect to that work.

\footnotetext{
${ }^{5} \mathrm{The} \mathrm{r} / \rho$ distribution was determined from 259 high grade orbital solutions from the Sixth Catalog of Orbits of Visual Binary Stars.
}

A different weighting process in Desidera et al. (2012) could show slighly different values for $\mathrm{d} \theta / \mathrm{d} t$ and $\mathrm{d} \rho / \mathrm{d} t$. Unfortunately, no information about this was detailed in Desidera et al. (2012). For example, graphically from his Figure 4, we

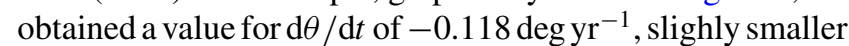
than our value. If we use this smaller value in our calculations, then the eccentricity vs. $z$ is in very good agreement with the work of Desidera et al. (2012).

\section{THE CIRCUMPRIMARY PLANETARY ORBIT}

We have showed that HD $106515 \mathrm{AB}$ is a gravitationally bound system. The dynamical study here presented uses several tests (some of them used for the first time for this system), which point out the gravitationally bound status. In this section, we will discuss how this scenario influences the planetary orbit around HD 106515A. The planet was spectroscopically monitored by Mayor et al. (2011) since 1998 using the CORALIE spectrograph, located at the 1.2-m EULER Swiss telescope at La Silla Observatory and over about 11 yrs using the spectrograph SARG at TNG Desidera et al. (2012).

Desidera et al. (2012) also computed the critical semimajor axis [calculated following Holman \& Wiegert (1999) prescriptions] and concluded that the planetary orbit is stable in a long term, possibly affected by periodic modulation due to KL $\left(P_{\mathrm{KL}}\right)$. The formula for the critical semimajor axis in Holman \& Wiegert (1999) only uses the outer binary eccentricity but not the planetary eccentricity, which is high for HD $106515 \mathrm{~b}$, and does not include any effect due to the mutual inclination.

Marmier et al. (2013) concluded that the KL, with a timescale of 3.3 Myr, is not suppressed by the much longer general relativistic timescale of $1.1 \mathrm{Gyr}$. To confirm this result, in this work, we have plotted the possible values of $P_{\mathrm{KL}}$ as a function of the outer semimajor axis and eccentricity obtained from a sample of about 27500 orbits. This was performed using all the input parameters used in the orbital calculation procedure within $95 \%(2 \sigma)$ interval confidence. Figure 6 was built for a planetary orbit inclination of $90^{\circ}$ (edge-on orbit), that is, for a planetary mass of 9.6 Jupiter 

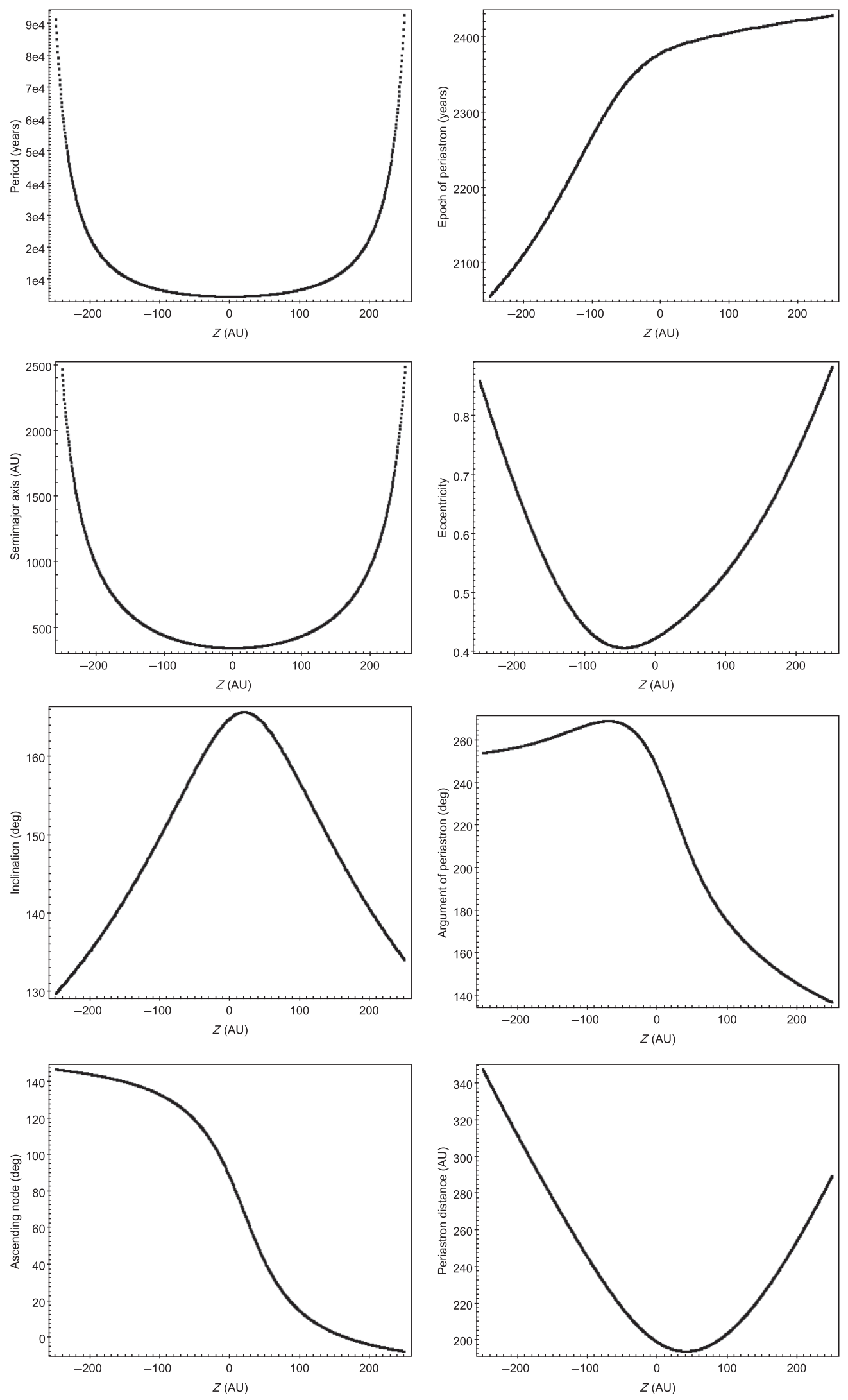

Figure 5. Distribution of orbital parameters vs. $z$. 


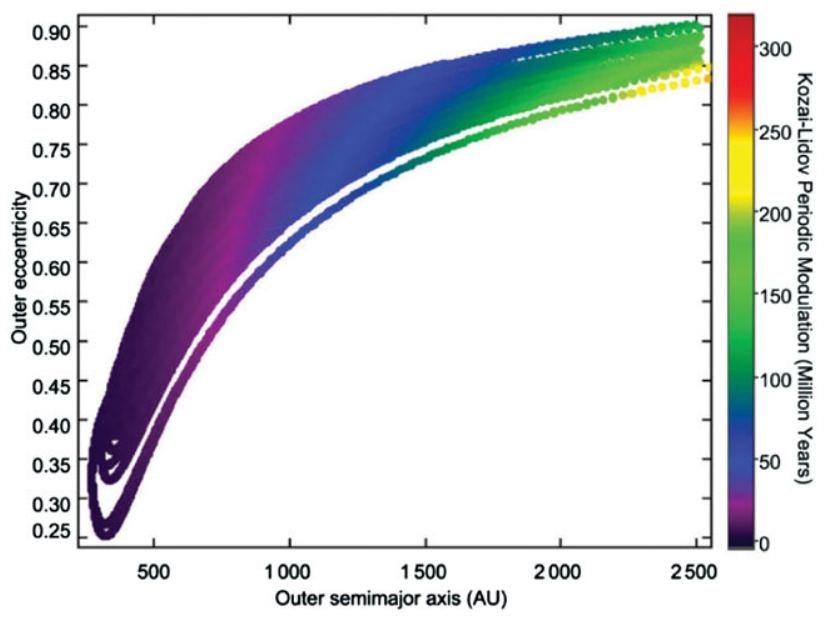

Figure 6. Kozai-Lidov periodic modulation for an edge-on planetary orbit (inclination $=90^{\circ}$ and a planetary mass of 9.6 Jupiter mass). Colour figure included in the electronic paper.

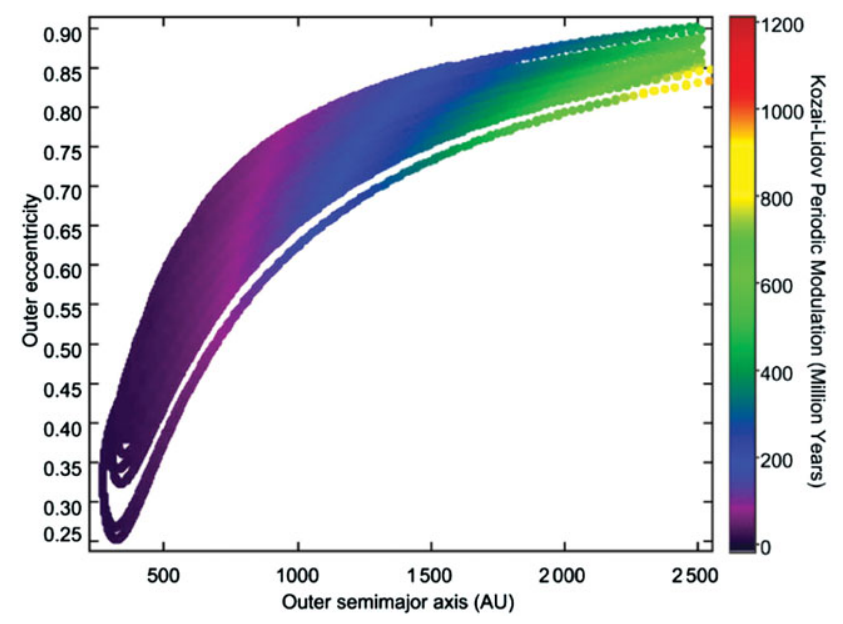

Figure 7. Kozai-Lidov periodic modulation for a nearly face-on planetary orbit (inclination $=0.2^{\circ}$ and a 'planetary' mass of $2.6 \mathrm{M}_{\odot}$ ). Colour figure included in the electronic paper.

masses. $P_{\mathrm{KL}}$ ranges from several millions years for small semimajor axis $(a<400 \mathrm{AU})$ to about $0.2-0.3 \mathrm{Gyr}$ for orbits with very high semimajor axis and eccentricities. The only scenario where $P_{\mathrm{KL}}$ could be suppressed by general relativistic precession period happens when the planet is in a nearly face-on orbit. Figure 7 plots $P_{\mathrm{KL}}$ for a planetary inclination of $0.2^{\circ}$ (when the mass of the 'planet' is of $2.6 \mathrm{M}_{\odot}$ ) with values for $P_{\mathrm{KL}}=1.2 \mathrm{Gyr}$ and very high semimajor axis and eccentricities. But this scenario is highly unlikely.

To activate the KL, the relative inclinations of planetary and binary orbital planes $(\Delta I)$ must be of $39.2^{\circ}$ Kozai (1962). But the angle needed to produce the eccentricity of HD $106515 \mathrm{~b}$, which is 0.57 , could be calculated approximately from the formula that relates the maximum eccentricity and the mutual inclination (Kozai (1962) and Lidov (1962)), from which we derive

$$
\Delta I=\arccos \sqrt{\frac{3\left(1-e_{\max }^{2}\right)}{5},},
$$

where $e_{\max }$ is the maximum eccentricity of the planetary orbit during the Kozai-Lidov periodic modulation. The planetary eccentricity gives the lower limit for $e_{\max }$ which yields a $\Delta I \geq 50.5^{\circ}$. But this expresion does not take into account the octopole and higher moments, nor the eccentricity of the HD 106515 AB.

The standard Kozai-Lidov mechanism (hereafter SKLM) has two major assumptions: (1) one of the component of the inner binary (in this case the planet) has negligible mass and therefore zero angular momentum; and (2) the outer orbit (that is HD $106515 \mathrm{AB}$ ) is circular. HD $106515 \mathrm{~b}$ is a very massive planet, nearly 10 Jupiter mass, so its angular momentum is $0.60 \mathrm{M}_{\odot}^{1 / 2} U A^{7 / 2} \mathrm{yr}^{-2}$. However, this is only about $0.01 \%$ of the total angular momentum ${ }^{6}$ and hence the first assumption may be considered valid.

The orbit of HD $106515 \mathrm{AB}$ is eccentric $(e \geq 0.42)$ and therefore the second assumption is not fulfilled. Naoz et al. (2011) and Naoz, Farr, \& Rasio (2012) showed that this situation leads to longer term octupole perturbations on the inner orbit (the so-called Eccentric Kozai-Lidov Mechanism, EKLM). The EKLM can lead to a dynamical behaviour significantly different from the SKLM. So, the inner orbit can flip between prograde and retrograde and in addition can reach extremely high values in eccentricity.

We use the REBOUND N-body numerical code (Rein \& Liu 2012) to perform numerical simulations. This code employs the high-order IAS15 integrator (Integrator and Adaptive Step-size control) based on a 15 th-order Gau $\beta$-Radau quadrature (Rein \& Spiegel 2015). We performed several simulations varying the initial mutual inclination $(\Delta I)$ with the time. Since the angular momentum of the planet is negligible with respect to the total angular momentum, the variation of the planetary inclination causes most of the variation of $\Delta I$.

The input orbital parameters for the simulations were

- for the outer orbit, that is, for the binary star, we consider those listed in the Table 5 in addition to the stellar masses of the Desidera et al. (2012) listed in Table 1.

- for the planetary orbit, we use the orbital parameters listed in The Extrasolar Planets Encyclopaedia web ${ }^{7}$ except for $i$ and $\Omega$, values that change in each simulations (see Table 6 describe in the next parragraph).

Figure 8 shows the evolution of the planetary orbit's eccentricity and inclination for different inclinations of the planet of $130^{\circ}, 100^{\circ}$, and $74^{\circ}$. These different inclinations yields to different initial mutual inclinatons of $\Delta I$ of $51.2^{\circ}, 68.7^{\circ}$ and

\footnotetext{
${ }^{6}$ The inner and outer orbital angular momentum were calculated using formulae (3) and (4) of Naoz et al. (2013).

${ }^{7} \mathrm{http}: / /$ exoplanet.eu/catalog/hd_106515_a_b/
} 

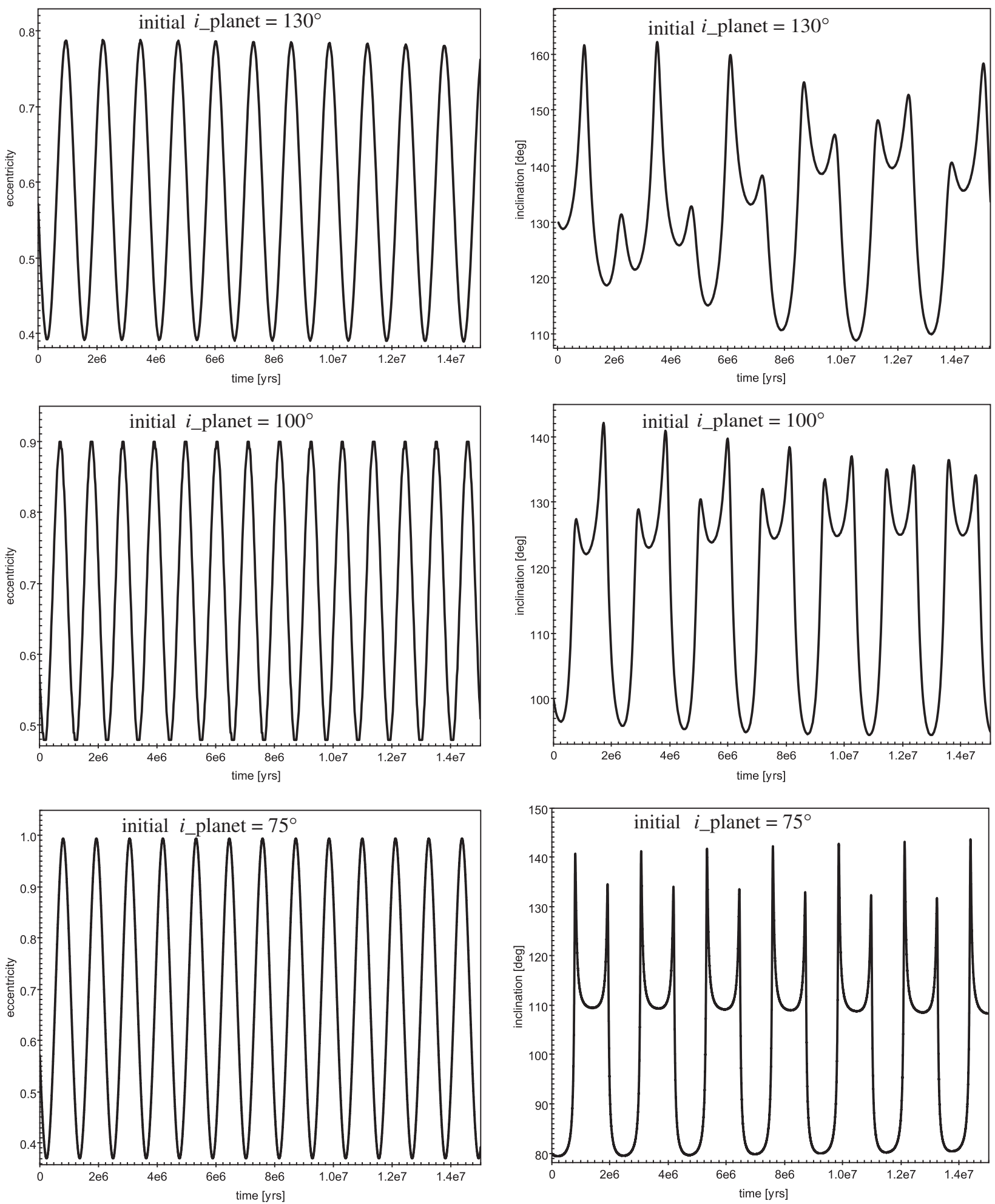

Figure 8. Evolution of the planetary orbit's eccentricity and inclination using REBOUND N-body numerical code. Simulations are showed for mutual inclination $(\Delta I)$ of $51.2^{\circ}, 68.7^{\circ}$, and $84.6^{\circ}$ with maximum eccentricity of $0.79,0.90$, and 0.995 and periodic oscillation of $1.3,1.05,1.13$ million yrs (oscillation are two times larger than the inclination). For $\Delta I=84.6^{\circ}$, the inclination flips from prograde to retrograde.

84.6 $6^{\circ}$ The results of these simulations (in addition to other simulation for $\Delta I=43.0$ or planetary inclination of $140^{\circ}$ ) are detailed in Table 6 which lists $\Delta I$, the planetary orbit's inclination $\left(i_{o}\right)$, the longitude of the ascending node $\left(\Omega_{o}\right)$, the periodic modulation of the eccentricity $\left(P_{\mathrm{ecc}}\right)$ and its lowerupper limits, and the periodic modulation of the inclination $\left(P_{\text {inc }}\right)$ and its lower-upper limits. The periodic modulation is expressed in millions of years and the periodic oscillations 
Table 6. The periodical modulation of the planetary eccentricity and inclination.

\begin{tabular}{lrrcccr}
\hline \hline $\begin{array}{l}\Delta I \\
\left({ }^{\circ}\right.\end{array}$ & $\begin{array}{c}i_{o} \\
\left.{ }^{\circ}\right)\end{array}$ & $\begin{array}{r}\Omega_{o} \\
\left({ }^{\circ}\right)\end{array}$ & $\begin{array}{c}P_{\text {ecc }} \\
\text { (Million years) }\end{array}$ & Eccentricity & $\begin{array}{c}P_{\text {inc }} \\
\text { (Million years) }\end{array}$ & $\begin{array}{c}\text { Inclination } \\
\left({ }^{\circ}\right)\end{array}$ \\
\hline 43.0 & 140 & 0 & 2.0 & $(0.26-0.59)$ & 3.6 & $(114-154)$ \\
51.2 & 130 & 180 & 1.3 & $(0.39-0.79)$ & 2.6 & $(109-162)$ \\
68.7 & 100 & 50 & 1.05 & $(0.48-0.90)$ & 2.1 & $(95-164)$ \\
84.6 & 80 & 100 & 1.13 & $(0.37-0.995)$ & 2.26 & $(80-144)$ \\
\hline \hline
\end{tabular}

for the inclination is exactly twice greater than for the eccentricity. For $\Delta I=84.6^{\circ}$, the inclination flips from prograde to retrograde ranges from $80^{\circ}$ to $144^{\circ}$. We also ran a simulation for $\Delta I=89.6^{\circ}$. At 0.78 million yrs, the planetary eccentricity increased to 0.9997 that yields a periastron distance of $0.00135772 \mathrm{AU}$ or $203658 \mathrm{~km}$. That is, HD 106515 b collided with HD 106515 A.

Summarising the planetary orbit is stable in the long term but modulated by periodic pertubations of about $2-3$ millions yrs due to EKLM.

\section{CONCLUSIONS}

We conducted a multi-purpose study on the stellar system HD 106515 AB. Using publicly available astrometric data, velocity and Hipparcos trigonometric parallax, we developed a dynamical study of this binary system.

We considered 133 measures reported in the WDS catalogue describing an orbital arc of about $21^{\circ}$ in a time base of 189 yrs. From this observational arc and using several tests (same of them used for the first time), we demonstrated that HD $106515 \mathrm{AB}$ is a bound system. We computed the orbital parameters of this binary for the first time. Considering the instantaneous relative position and velocity, we estimated the most probable orbital solution with an orbital period of about $4800 \mathrm{yrs}$, a semimajor axis of $\sim 350 \mathrm{AU}$, an inclination of $\sim 165^{\circ}$ and an eccentricity about 0.42 .

We also found evidence of the influence of the binary orbit on the planetary one present in the HD 106515 A star. The critical semimajor axis ensures the stability of the planetary orbit. The ratio between Kozai and general relativistic precession periods $(\ll 1)$ suggests that the Kozai mechanism could be perturbing the planetary orbit.

Our study of the dynamical evolution of the planetary orbit using REBOUND N-body numerical code shows periodic oscillation for the eccentricity larger than those calculated using the classical formula. This effect is greater for low $\Delta I$. The reason being neither the octopole and higher moments, nor the eccentricity of the HD $106515 \mathrm{AB}$ are taken into account for this formula. For example, considering the $\Delta I$ values listed in Table 6, the maximum eccentricities calculated are $0.33,0.59,0.88$, and 0.993 . For the last two values of $\Delta I$, our maximum eccentricities are very close.

We determined that $\Delta I$ must be greater than $50.5^{\circ}$, which yields a planetary eccentricity of 0.57 . On the other hand, the
REBOUND simulations show that higher $\Delta I$ leads to more eccentric orbits.

Marmier et al. (2013) estimated a $P_{\mathrm{KL}}$ of $3.3 \mathrm{Myr}$ assuming an outer eccentricity with a median value of a distribution $f(e)=2 e$. Our eccentricity periodical modulation is smaller than that value.

In addition to all above exposed, the work here presented try to be an example of how using publicly available data and high precission measurements, dynamical studies of longperiod binary system with exoplanets can be performed in the pre-GAIA era.

\section{ACKNOWLEDGEMENTS}

Rafael Barrena acknowledges financial support from the Spanish Ministry of Economy and Competitiveness (MINECO) under the 2011 Severo Ochoa Program MINECO SEV-2011-0187.

This research work makes use of data from the Two Micron All Sky Survey (2MASS), which is a joint project of the University of Massachusetts and the Infrared Processing and Analysis Center/California Institute of Technology, funded by the National Aeronautics and Space Administration and the National Science Foundation. The study here presented also uses the Washington Double Star Catalog, maintained at the U.S. Naval Observatory and the SIMBAD astronomical database and VizieR astronomical catalogues service, both maintained and operated by the Center de Données Astronomiques de Strasbourg (http://cdsweb.u-strasbg.fr/).

The authors thank Josefina Ling, Henry Zirm, and Frank Smith for their respective reviews of this work.

\section{REFERENCES}

Adams, E. R., et al. 2012, AJ, 144, 42

Adams, W. S., Joy, A. H., Humason, M. L., \& Brayton, A. M. 1935, ApJ, 81, 187

Benavides, R., et al. 2010, JDSO, 6, 30

Bessell, M. S. 1990 PASP, 102, 1181

Bressan, A., et al. 2012, MNRAS, 427, 127

Burnham, S. W. 1913, Measures of Proper Motion Stars Made with the 40-inch Refractor of the Yerkes Observatory in the Years 1907 to 1912 (Washington, D.C.: Carnegie Institution of Washington)

Burningham, B., et al. 2009, MNRAS, 395, 1237

Close, L. M., Richer, H. B., \& Crabtree, D. R. 1990, AJ, 100, 1968

de Lalande, J.-J. 1831, MmRAS, 4, 165

Desidera, S., Gratton, R. G., Lucatello, S., Claudi, R. U., \& Dall, T. H. 2006, A\&A, 454, 553

Desidera, S., et al. 2004, A\&A, 420, 683 
Desidera, S., et al. 2012, A\&A, 546, 108

Duflot, M., Figon, P., \& Meyssonnier, N. 1995, A\&AS, 114, 269

Gould, A., \& Chanamé, J. 2004, ApJS, 150, 455

Grocheva, E., \& Kiselev, A. 1998, ASPC, 145, 15

Hartkopf, W. I., \& Mason, B. D. 2011a, AJ, 122, 3472

Hartkopf, W. I., \& Mason, B. D. 2011b, Catalog of Rectilinear Elements, http://www.usno.navy.mil/USNO/astrometry/ optical-IR-prod/wds/lin1

Hauser, H. M., \& Marcy, G. W. 1999, PASP, 111, 321

Herschel, J. F. W. 1829, MmRAS, 3, 177

Herschel, J. F. W. 1870, MmRAS, 38, 1

Hog, E., et al. 2000, A\&A, 355L, 27

Holman, M. J., \& Wiegert, P. A. 1999, AJ, 117, 621

Houk, N., \& Swift, C. 1999, Michigan Catalogue of TwoDimensional Spectral Types for the HD Stars, Vol. 5 (Ann Arbor: Department of Astronomy, University of Michigan)

Jiang, Y.-F., \& Tremaine, S. 2010, MNRAS, 401, 977

Jones, E. M. 1972, ApJ, 173, 671

Kiselev, A. A. 1989, Theoretic Foundations of Photographic Astrometry (Moscow: Nauka)

Kiselev, A. A., \& Kiyaeva, O. V. 1980, SvA, 24, 706

Kiselev, A. A., Kiyaeva, O. V., Romanenko, L. G., \& Gorynya, N. A. 2012, ARep, 56, 524

Kozai, Y. 1962, AJ, 67, 591

Lidov, M. L. 1962, P\&SS, 9, 719

Lodieu, N., et al. 2014, A\&A, 569, 120

Maíz-Apellániz, J. 2006, AJ, 131, 1184

Mamajek, E. E., et al. 2013, AJ, 146, 154
Marmier, M., et al. 2013, A\&A, 551, 90

Mason, B. D., Wycoff, G. L., Hartkopf, W. I., Douglass, G. G., \& Worley, C. E. 2001, AJ, 122, 3466

Mayor, M., et al. 2011, A\&A, submitted, arXiv:1109.2497M

Naoz, S., Farr, W. M., Lithwick, Y., Rasio, F. A., \& Teyssandier, J. 2011, Nature, 473, 187

Naoz, S., Farr, W. M., Lithwick, Y., Rasio, F. A., \& Teyssandier, J. 2013, MNRAS, 431, 2155

Naoz, S., Farr, W. M., \& Rasio, F. A. 2012, ApJL, 754, L36

Nuget, R. L., \& Iverson, E. W. 2015, JDSO, 11, 21

Oscoz, A., et al. 2008, SPIE, 7014, 701447

Pickles, A., \& Depagne, E. 2010, PASP, 122, 1437

Rein, H., \& Liu, S.-F. 2012, A\&A, 537, A128

Rein, H., \& Spiegel, D. S. 2015, MNRAS, 446, 1424

Rica, F. M. 2011, JDSO, 7, 254

Rica, F. M. 2012, JDSO, 8, 260

Rica, F. M., Barrena, R., Vázquez, G., Henríquez, J. A., \& Hernández, F. 2012, MNRAS, 419, 197

Roell, T., Neuhäuser, R., Seifahrt, A., \& Mugrauer, M. 2012, A\&A, 542,92

Santos, N. C., et al. 2013, A\&A, 556A, 150

South, J., \& Herschel, J. 1824, RSPT, 114, 1

Struve, F. G. W. 1837, AN, 14, 249

Torres, G. 1999, PASP, 111, 169

van den Bos, W. H. 1932, CiUO, 86, 261

van Leeuwen, F. 2007, A\&A, 474, 653

Weinberg, M. D., Shapiro, S. L., \& Wasserman, I. 1987, ApJ, 312, 367 